\title{
Uma Abordagem Gamificada para Prevenção do uso de Drogas com Adolescentes
}

\author{
Camila D. de Medeiros, Anderson M. Fernandes, Eduardo F. Damasceno ${ }^{1}$ \\ ${ }^{1}$ Universidade Tecnológica Federal do Paraná (UTFPR) - Cornélio Procópio - PR \\ camiladutram7@,hotmail.com; mine@,fau.com.br ; damasceno@utfpr.edu.br
}

\begin{abstract}
This meta-paper proposes a RPG-liked (Role Playing Game) game as alternative to assist in the campaign to prevent drugs-use. It is concluded that the potential of the game characterized for this community makes up a greater awareness than other approaches presented.
\end{abstract}

Resumo. Este trabalho apresenta a proposta de um jogo digital no formato de RPG (Role Playing Game) como forma alternativa auxiliar na campanha de prevenção ao uso de drogas. Conclui-se que a potencialidade do jogo caracterizado para esta comunidade perfaz uma maior conscientização do que as demais abordagens apresentadas.

\section{Introdução}

A adolescência é uma fase da vida em que as descobertas são um fator relevante para formação conhecimento, da afirmação de caráter e identificação do indivíduo na sociedade [Williams, Meyer \& Pechansky, 2007]. Esta fase é comumente relatada como um período de preocupações para os pais, principalmente no que tange a questão de uso de drogas. Sendo uma fase caracterizada como uma fase de procura, de descobertas, na qual os adolescentes dão muita importância aos seus grupos, seus relacionamentos, e terminam por entrar em conflito consigo mesmos e com a família quando assumem participar de novos espaços e novos comportamentos. Esses espaços muitas vezes os tornam mais vulneráveis a situações externas, tais como o consumo de drogas, delinquência e condutas sexuais de risco [dos Santos, et al., 2011].

Em diversos trabalhos encontrados na literatura sobre o consumo ao consumo de drogas ilícitas no Brasil, destacam-se os números relativo ao primeiro na adolescência e partindo para a fase da juventude. Em um estudo transversal sobre o tema, foi apresentado que de 196 sujeitos pesquisados de faixa etária entre 14 a 16 anos, aproximadamente $18 \%$ já experimentaram algum tipo de droga ilícita (maconha, crack, ou anfetaminas diversas) [Tavares, Béria, \& Lima, 2001].

Diversas abordagens vem sendo aplicadas para a repressão às Drogas, dentre elas se destacam o uso da força policial e militar pelo governo, no intuito de coibir e interromper o tráfico de drogas [Williams, Meyer \& Pechansky, 2007]; Políticas públicas para internação compulsória para tratamento da dependência [Baus, Kupek \& Pires, 2002]; Aproximação e debate na escola com intuito de esclarecer e realizar o sensibilização pela prevenção ao ato [dos Santos, et al., 2011].

O objetivo desta aproximação, muito comum nas campanhas publicitárias, é instruir crianças e adolescentes a rejeitar as drogas, principalmente as ilegais, e convencer usuários ocasionais a pararem o consumo totalmente. Todavia, é percebido as campanhas que não embarcaram êxito usufruíram-se de conceitos e problemática do senso comum, já conhecidas pelos jovens [Hornik \& Jacobsohn, 2007].

Acredita-se que a falta de êxito das campanhas é atribuída a: uso de linguagem inadequada ao público jovem, o que dificulta a compreensão das mensagens [Veronese \& Silva, 2009]. Portanto, uma abordagem mais adequada a este público poderá lograr maior sucesso, como por exemplo, a abordagem por jogos digitais, ou games. 
Está cada vez mais comum se observar o baixo interesse e a pouca motivação por parte dos adolescentes e jovens no que diz respeito à formação tradicional de como o conhecimento e a informação vem sendo transposta para eles [Nascimento, 2013]. Por outro lado, quando se tem um olhar mais atento ao entorno desses mesmos jovens, observa-se o grande fascínio e envolvimento deles com os jogos de computador.

Desta forma, este trabalho visa apresentar um jogo digital para auxiliar na prevenção ao uso de drogas por adolescentes e jovens, neste contexto a abordagem escolhida foi da análise comportamental do usuário no intuito de dar mais subsídios aos terapeutas e assistentes sociais a tomarem decisões sobre a iminência do uso de drogas no meio escolar.

\section{O Processo de Gamificação}

O processo de Gamificação pode ser entendido como o uso de metáforas de jogos em contextos específico, visando influenciar um comportamento, melhorar a motivação e incrementar o engajamento do seu jogador em uma atividade simulada.

É possível compreender pelo menos duas ideias centrais neste contexto [Barnes, 2013]: 1) a influência que os games e elementos de games estão moldando o cotidiano das pessoas e suas interação com os novos dispositivos; 2) os games e os elementos de games produzem comprovadamente estados de experiências desejáveis, podendo motivar jogadores a permanecerem engajados em atividades com durações e intensidades sem precedentes.

Discute-se muito sobre a relação do contexto (1) apresentado, pois há uma divergência entre a construção de uma informação e o cultivo de um comportamento relacionado. E para o contexto (2) a discussão está relacionada a forma como um jogador é afetado, sendo que na ideia (1) o jogador é afetado pelo meio e pelo contexto no qual está inserido fazendo com que seu comportamento mude e na ideia (2) o jogador é auto-afetado a partir de emoções e incentivos que estão em acordo com seus próprios valores [Deterding, 2011].

Então, assumiu-se para este trabalho o conceito mais difundido sobre a gamificação, "um processo pensamento de games e mecânicas de games para engajar usuários e resolver problema" [Kapp, 2012].

Um processo de gamificação tende a levar a informação de forma mais natural e mais afixada no contexto do jogador, pois este aprende sem perceber, de forma simplista e desenvolve uma habilidade, antes apenas almejada pela explanação, agora é sensibilizada pelo game. Neste processo foi observado alguns requisitos que um jogo deveria cumprir [Zichermann \& Cunningham, 2011]: a) Narrativa; b) Conflito; c) Recompensa; d) Níveis de dificuldade, e; e) Regras.

Os pesquisadores, terapeutas e clínicos, em todo o mundo, têm grande interesse em utilizar todas as estratégias que possam aumentar as chances de sucesso dos programas de tratamento oferecidos para jovens usuários de drogas [Adade \& Monteiro, 2013].

Uma ferramenta utilizada com adolescentes que pode contribuir no tratamento e na prevenção de problemas psicológicos e sociais é o jogo terapêutico. Exemplos desses tipos de instrumento são o "Jogo da Escolha" [Williams, Meyer, \& Pechansky, 2007] e o "Jogo da Onda" [Rebello, Monteiro \& Vargas, 2001], os quais visam educar os jovens a respeito do uso de substâncias psicoativas, além de treinar habilidades para evitar lapsos ou recaídas [Araujo et al., 2011].

Entre as modalidades de jogos possíveis de serem utilizadas no tratamento de adolescentes, destaca-se o Role-Playing Game (RPG) ou "jogo de interpretação de personagens", que é uma modalidade de jogo na qual os participantes assumem papéis de personagens, criando narrativas de modo colaborativo. Existe um sistema de regras predeterminado, mas há espaço para a improvisação [Straczynski \& Sprange, 2006]. 
O "Role-Playing Game Desafios" é um jogo de cartas fundamentado na Terapia CognitivoComportamental que tem como objetivo treinar habilidades de adolescentes para enfrentar as situações de risco para o uso de substâncias psicoativas, bem como corrigir crenças distorcidas relacionadas ao efeito das drogas. É "um instrumento de diagnóstico, intervenção e autoorientação que constitui uma espécie de objeto gráfico múltiplo e interativo com potencial terapêutico e de pesquisa" (Timm, 2009).

Nota-se que o RPG tende a ser a melhor ferramenta no tratamento de prevenção de drogas, pois devido às características contidas nessa modalidade de jogo, um leque de opções podem ser abordados. Porém, vale ressaltar que um RPG nem sempre oferece a saída necessária para determinada situação, sendo o jogador obrigado a seguir regras predeterminadas, não realizando uma opção de sua escolha, atrapalhando os próprios resultados.

\section{A Sistemática do Jogo}

De forma a manter o usuário recebendo diversas informações sobre o uso de drogas, o objetivo do jogo também é de compreender as ações e reações que o usuário tem e sua percepção sobre o que é uma droga (lícita e ilícita).

Destarte, foi elaborado um mapa de caminhos possíveis que o usuário pode tomar a cada fase do jogo. Estes caminhos ligam uma fase a outra e foram desenvolvidos de acordo com a teoria de redução de danos [Marlatt \& Bueno, 1999]. A cada nova situação apresentada ao usuário ele tem duas opções: uma boa e outra ruim, todavia, a ruim depende diretamente de sua visão de mundo, e a cada escolha ruim ele é levado a uma nova situação um pouco menos ruim (redução do dano). Assim, se na situação ruim ele escolher a opção de melhor impacto (boa) ele será recolocado em outra fase com situação boa, este exemplo pode ser melhor visto na ilustração da Figura 1.

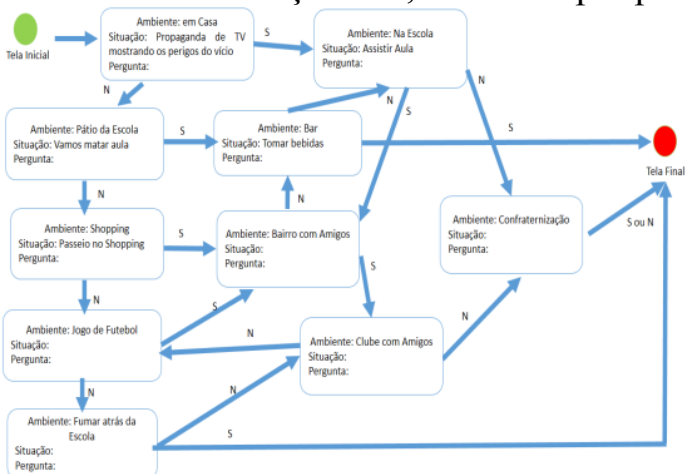

a)Fases do Jogo

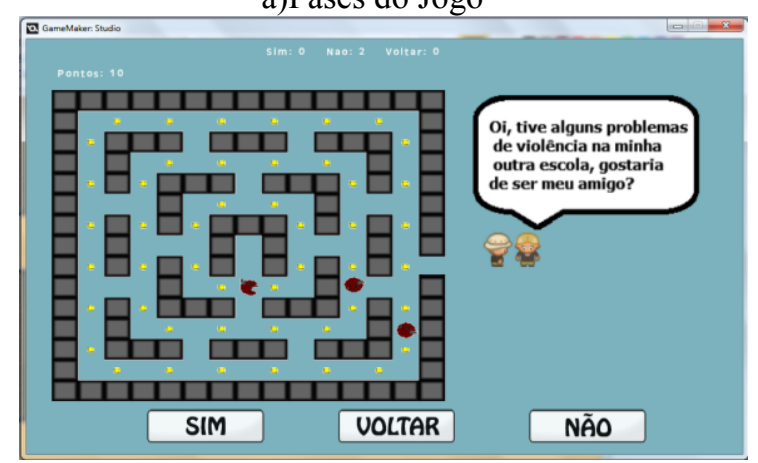

c)Atividade óculo-Manual

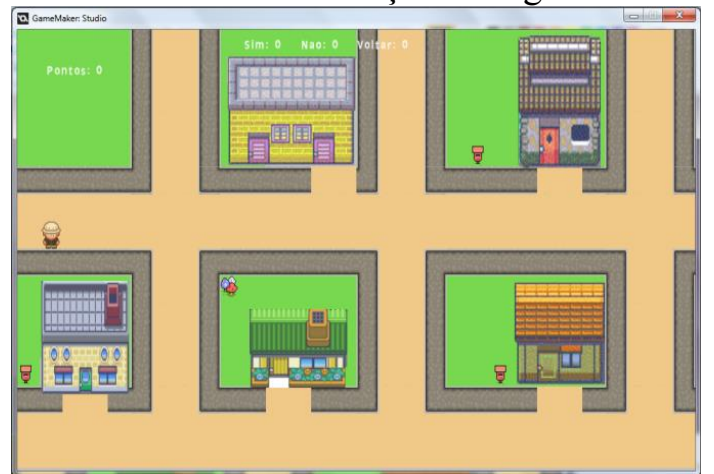

b)Tela do Jogo - Passeio e Exploração

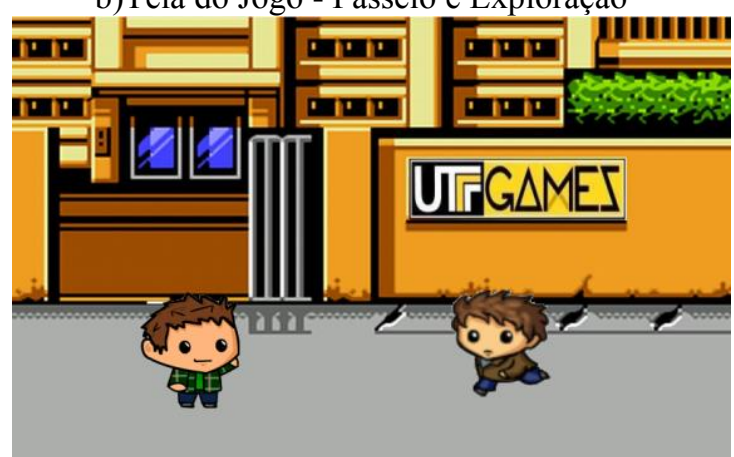

d)Tela de evidenciando a convivência

Figura 1. Fluxograma das Situações do Jogo

\section{Avaliação e Resultados}

No intuito de promover a melhoria do processo e lograr sucesso para a prevenção do uso de drogas por adolescentes foi realizado uma avaliação do software (jogo) quanto aos quesitos de interface 
(Battaiola et. al, 2002), jogabilidade (Cuperschmid, 2008) e comunicabilidade (Prates \& Souza, 2013) da mensagem sobre o uso de drogas com uma comunidade escolar (alunos e professores), o método de aferição foi um questionário em que cada pergunta estava pautada sua resposta na escala Likert, de forma que os sujeitos da pesquisa pudessem ponderar as suas impressões sobre o jogo.

A recepção do jogos pela comunidade escolar teve dois momentos distintos, um inicial pelos docentes, e outro mais acalorado pelos alunos. Os alunos mostraram-se interessados por poder jogar um jogo e ainda aprender. E de outra forma os alunos ao jogar perceberam que estavam livres para escolher qualquer opção e sentiram-se livres para evidenciar suas dúvidas sobre o uso de drogas. E após a tabulação dos dados, percebeu-se que as informações obtidas estão tendendo para a concordância incondicional de todas as perguntas realizadas, podendo este fato ser atribuído ao contato com uma tecnologia ou alguma falha no método escolhido para a pesquisa. Com isto, não há como fazer uma análise apurada das informações, apenas podemos ponderar algumas afirmativas e constatar que a aplicação pode ser ótima aliada ao processo de ensino, quando aplicada de forma auxiliar.

\section{Contribuições e Considerações Finais}

A partir do exposto apresentado, nota-se a falha de comunicação que existe entre medidas preventivas e adolescentes em relação ao consumo de drogas. É sabido que normalmente nessa fase, considerada de procura e descoberta, ocorra o primeiro contato com algum tipo de droga ilícita.

Como medida de prevenção, pesquisas apontam que o uso de jogos digitais juntamente com o estilo de jogo RPG tende a ser uma importante ferramenta, devido a características de aprendizagem e motivação, levando o estudo à área educacional. E observando o crescimento da indústria de software de jogos eletrônicos uma a aplicação voltada para que seus jogadores tenham horas de lazer nas quais tenham os mais completos e diferentes tipos de desafios característicos dos jogos, sejam eles eletrônicos ou não, tendem a influenciar positivamente, e desta forma o objetivo da prevenção poderá ser obtido.

Ademais, os jogos computacionais permitem a criação de um nicho mercadológico que propicia o desenvolvimento de software e fomenta o desenvolvimento de pesquisas no meio acadêmico.

\section{Referencias}

ADADE, M., \& MONTEIRO, S. (2013). Educação sobre drogas: uma proposta orientada pela redução de danos. Educação e Pesquisa.

ARAUJO, R; OLIVEIRA, M; CEMI, J. (2011). Desenvolvimento de Role-Playing Game para Prevenção e Tratamento de Dependência de Drogas da Adolescência. Psicologia: Teoria e Pesquisa, São Paulo, vol. 27, n. 3, p 347-356, jul-set, 2011.

BARNES, J “3 Keys To Gamification For Education," InformationWeek, 2013.

BATTAIOLA, A. L., ELIAS, N. C., DOMINGUES, R. G., ASSAF, R., \& RAMALHO, G. L. Desenvolvimento da Interface de um Software Educacional com base em Interfaces de Jogos. IHC Simpósio sobre Fatores Humanos em Sistemas Computacionais, 2002.

BAUS, J., KUPEK, E., \& PIRES, M. (2002). Prevalência e fatores de risco relacionados ao uso de drogas entre escolares. Rev Saúde Pública, 36(1), 40-6.

CORRÊA, E. S. (2010). Aprende-se com videogames? Com a palavra, os jogadores. Dissertação (mestrado) - Universidade Federal de Santa Catarina, Centro de Ciências da Educação, Programa de Pós-Graduação em Educação, Florianópolis, 2010.

CUPERSCHMID, A.R.M. "Heurísticas de jogabilidade para jogos de Computador." Dissertação de Mestrado - Universidade Estadual de Campinas, Campinas (2008). 
DETERDING, S. "Situated motivational affordances of game elements : A conceptual model," in Congress in Computer Human Interface - CHI, 2011, pp. 3-6.

HORNIK, R., \& JACOBSOHN, L. (2007). The best laid plans: disappointments of the National Youth Anti-Drug Media Campaign. LDI issue brief, 14(2), 1-4.

KAPP, K.M., "The Gamification of learning na Instruction", 1st ed. San Francisco,CA: John Wiley, 2012, p. 243.

MARLATT, G. A, \& BUENO, D. (1999) Redução de danos: estratégias práticas para lidar com comportamentos de alto risco. Artes Médicas.

NASCIMENTO, V. T. D. (2013). O adolescente em conflito com a lei: um olhar acerca da Fundação de Atendimento Socioeducativo-FASE, de Porto Alegre.

PRATES, R. O \& SOUZA, C.S. (2013) "Extensão do teste de comunicabilidade para aplicações multi-usuário." Cadernos do IME-Série Informática 13: 51-62.

SANTOS, E. O., Maria de Fátima, S., da Silva Kauark, F., \& Manhães, F. C. (2011). Abordagem sobre a prevenção das drogas no contexto escolar. InterSciencePlace, 1(17).

STRACZYNSKI, J. M., \& SPRANGE, M. (2006). Role Playing Game. Mongoose Publishing. TAVARES, B. F.; BÉRIA, J. U., \& LIMA, M. S. (2001). Prevalência do uso de drogas e desempenho escolar entre adolescentes. Rev. Saúde Pública, 35 (2), 150-8.

TIMM, M. (2009) Um objeto gráfico múltiplo e interativo: potencial terapêutico e de pesquisa. Ver. Pisiquiatra, Rio Grande do Sul, p 84.

VERONESE, J.R.P. \& SILVA, R.L. (2009) "O acesso à cultura, informação e entretenimento e as medidas de prevenção previstas no estatuto da criança e do adolescente". Revista Sequência 30(59): 299-326.

VIANA, C;. (2005). O Lúdico e a Aprendizagem na Cibercultura: Jogos Digitais e Internet no Cotidiano Infantil, Tese (Doutorado em Ciências da Comunicação)-Escola de Comunicações e Arte, Universidade de São Paulo, São Paulo.

WILLIAMS, A. V., MEYER, E., \& PECHANSKY, F. (2007). Desenvolvimento de um jogo terapêutico para prevenção da recaída e motivação para mudança em jovens usuários de drogas. Psicologia: Teoria e Pesquisa, 23(4), 407-414.

ZICHERMANN, G \& CUNNINGHAM, C.(2011) "Gamification by Design: Implementing Game Mechanics in Web and Mobile Apps". O'Reilly Media Inc., p. 342. 